\title{
Using single quantum states as spin filters to study spin polarization in ferromagnets
}

\author{
Mandar M. Deshmukh and D. C. Ralph \\ Laboratory of Atomic and Solid State Physics, Cornell University, Ithaca, NY 14853
}

(November 15, 2018)

\begin{abstract}
By measuring electron tunneling between a ferromagnet and individual energy levels in an aluminum quantum dot, we show how spin-resolved quantum states can be used as filters to determine spin-dependent tunneling rates. We also observe magnetic-field-dependent shifts in the magnet's electrochemical potential relative to the dot's energy levels. The shifts vary between samples and are generally smaller than expected from the magnet's spin-polarized density of states. We suggest that they are affected by field-dependent charge redistribution at the magnetic interface.
\end{abstract}

PACS numbers: 73.23.Hk, 75.50.Cc, 75.70.Cn

Quantum dots are useful for studying electron spins, because they allow individual spin-resolved states to be examined in detail. Previous experiments have probed spin physics within several types of quantum dots: semi-

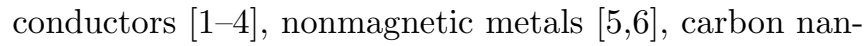
otubes [7], and ferromagnets [8]. Here we use the individual spin-resolved energy levels in a quantum dot to investigate the physics of a bulk magnetic electrode. The spin polarization in the magnet affects electron tunneling via the dot levels in two ways. First, tunneling rates are different for spin-up and spin-down electrons; we demonstrate how the tunneling polarization can be measured by using quantum-dot states as spin filters [9]. Second, as a function of magnetic field, the electrochemical potential of the magnetic electrode shifts relative to the energy levels in the dot. Previously, tunneling polarizations 10] and electrochemical shifts 11] have been measured by other techniques in larger devices having continuous densities of electronic states. By probing at the level of single quantum states, we are able to compare both effects in one device. We also achieve more precise measurements of the electrochemical shifts which demonstrate that they are not determined purely by the bulk properties of the magnet, as has been assumed previously [11,12].

Our quantum dot is an $\mathrm{Al}$ particle, $5-10 \mathrm{~nm}$ in diameter, connected by $\mathrm{Al}_{2} \mathrm{O}_{3}$ tunnel junctions to an $\mathrm{Al}$ electrode on one side and a cobalt or nickel electrode on the other (Fig. 1, inset). We use an Al particle to minimize spin-orbit coupling, so that electronic states within the particle are to a good approximation purely spin-up or spin-down 5.6. Device fabrication is done following the recipe in [5], except that in the final step we deposit $80 \mathrm{~nm}$ of magnetic Co or $\mathrm{Ni}$ at a pressure of $\sim 2 \times 10^{-7}$ torr to form the second electrode. We conduct tunneling measurements in a dilution refrigerator, using filtered electrical lines that provide an electronic base temperature of approximately $40 \mathrm{mK}$. Beyond a threshold voltage determined by the charging energy, electron tunneling via individual quantum states in the $\mathrm{Al}$ particle produces discrete steps in the $I-V$ curve $[5]$ or equivalently peaks in $d I / d V$ vs. $V$ (Fig. 1). The sign of bias refers to the sign applied to the $\mathrm{Al}$ electrode. Figure 2 shows how the energy levels in the particle undergo Zeeman spin-splitting as a function of magnetic field ( $B$, applied in the plane of the nitride membrane) 5 . The Co-lead sample also exhibits nonlinearities for $B<0.3 \mathrm{~T}$, possibly associated with magnetic-domain rotation.
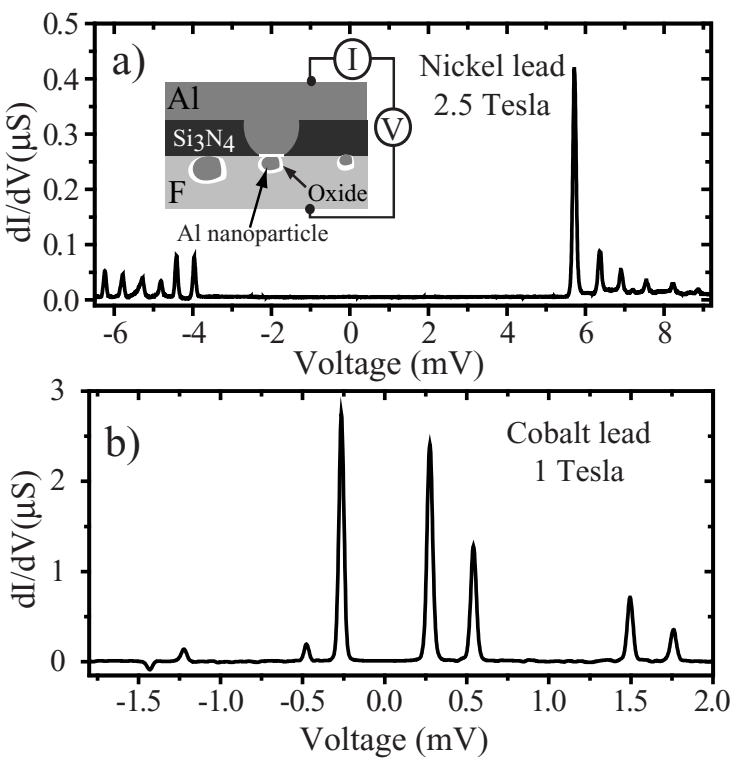

FIG. 1. (inset) Cross-sectional device schematic. (a) Differential conductance vs. $V$ for device Ni\#1 with one $\mathrm{Ni}$ electrode, and (b) for device Co\#1 with one Co electrode. Magnetic fields are applied to cause Zeeman splitting of the spin-up and spin-down resonances.

Before we turn to our main results, we note some experimental details. In order to convert the measured voltages of the resonances to energy, one must correct for the capacitive division of $V$ across the two tunnel junctions. For a tunneling transition across the nonmagnetic (N) junction, this is accomplished by multiplying $V$ by $e C_{F} /\left(C_{N}+C_{F}\right)$ and for the ferromagnetic (F) junction by $e C_{N} /\left(C_{N}+C_{F}\right)$, where $C_{N}$ and $C_{F}$ are the two junction capacitances. The capacitance ratio can be de- 
termined by comparing the voltage for tunneling through the same state at positive and negative $V$ [5]. We must also understand whether a resonance corresponds to a threshold for an electron tunneling on or off the particle, and across which tunnel junction. The transitions which correspond to tunneling between the particle and the $\mathrm{Al}$ electrode can be identified by the presence of a shift in their $V$ positions as the $\mathrm{Al}$ electrode is driven from superconducting to normal by a magnetic field, and by the effect of the superconducting density of states (DOS) on the resonance shape [5]. The sign of $V$ then determines whether an electron is tunneling on or off the particle. For the sample (Ni\#1) shown in Figs. 1(a) and 2(a,b), the transitions at positive $V$ correspond to tunneling first from the dot to the $\mathrm{Al}$ electrode, with $e C_{F} /\left(C_{N}+C_{F}\right)=$ $(0.42 \pm 0.02)$ e. For the sample (Co\#1) in Figs. 1(b) and 2(c), at positive $V$ electrons are initially tunneling from the Co electrode to the particle, and $e C_{N} /\left(C_{N}+C_{F}\right)=$ $(0.44 \pm 0.01)$ e.

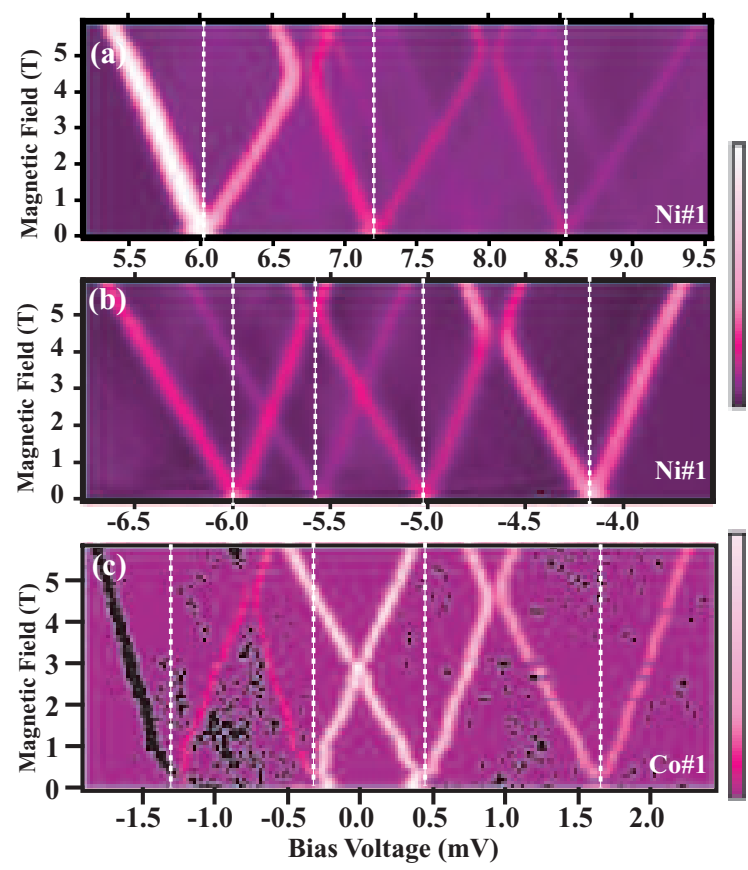

FIG. 2. $d I / d V$ vs. voltage and magnetic field for $(\mathrm{a}, \mathrm{b})$ device $\mathrm{Ni} \# 1$ and (c) Co\#1. The scales extend from 0 to $(\mathrm{a}, \mathrm{b})$ $0.2 \mu \mathrm{S}$ and (c) $2 \mu \mathrm{S}$. White indicates $d I / d V$ values beyond the scale maximum, and in (c) black indicates negative values.

We will now analyze how the currents carried by individual states allow measurements of spin-dependent tunneling rates. The resistances of our tunnel junctions are sufficiently large (at least $1 \mathrm{M} \Omega \gg h / e^{2}$ ) that transport can be modeled by sequential tunneling 13 14]. The analysis takes a particularly simple form when the offset charge [13] has a value that permits tunneling at a low value of $V$ so that only a single orbital state on the quantum dot contributes to current flow near the tunneling threshold [14,15]. This is the case for sample Co\#1; the thresholds for more complicated non-equilibrium tunnel- ing processes, involving the lowest-energy even-electron excited state [9], are $V<-5.8 \mathrm{mV}$ or $V>4.2 \mathrm{mV}$ at $B=0$ in this sample. In general the simple equilibrium tunneling regime can be achieved for any nanoparticle device made with a gate electrode so that the offset charge can be adjusted [15]. In Fig. 3(a) we show the $I$ - $V$ curve for sample Co\#1 with $B=1 \mathrm{~T}$ to Zeeman-split the resonances. The first step in current for either sign of $V$ corresponds in this sample to an electron tunneling through only a spin-up (majority-spin) state. The sequential tunneling theory [14] predicts that these two currents should have identical magnitudes,

$$
I_{1+}=\left|I_{1-}\right|=e \gamma_{\uparrow} \gamma_{N} /\left(\gamma_{\uparrow}+\gamma_{N}\right),
$$

where $\gamma_{\uparrow}$ is the bare tunneling rate between the magnet and the spin-up state, and $\gamma_{N}$ is the tunneling rate to the $\mathrm{Al}$ electrode. The fact that the steps do have the same magnitude confirms that electrons are tunneling via just one state. When $|V|$ is increased to permit tunneling through either the spin-up or spin-down state, the predicted values for the total current, using the methods in [13, 14, are for positive and negative $V$,

$$
\begin{gathered}
I_{2+}=\frac{e \gamma_{N}\left(\gamma_{\uparrow}+\gamma_{\downarrow}\right)}{\gamma_{N}+\gamma_{\uparrow}+\gamma_{\downarrow}}, \\
\left|I_{2-}\right|=\frac{2 e \gamma_{N}}{1+\gamma_{N} / \gamma_{\uparrow}+\gamma_{N} / \gamma_{\downarrow}} .
\end{gathered}
$$

We have made use of time-reversal symmetry which requires that the tunneling rates from the nonmagnetic electrode to both Zeeman-split states should be the same. This has been verified in a previous experiment 15]. We have also neglected spin relaxation based on experimental limits of relaxation rates slower than $5 \times 10^{7} \mathrm{~s}^{-1}$ in $\mathrm{Al}$ particles with $\mathrm{Al}$ electrodes [15], much slower than the tunneling rates. Equations (1)-(3) can be inverted to determine $\gamma_{N}, \gamma_{\uparrow}$, and $\gamma_{\downarrow}$ from $I_{1+}, I_{2+}$, and $I_{2-}$ (Fig. 3(c)). The resulting tunneling polarization, $\left(\gamma_{\uparrow}-\gamma_{\downarrow}\right) /\left(\gamma_{\uparrow}+\gamma_{\downarrow}\right)$, is positive (Fig. 3(d)), meaning that the tunneling rate for spin-up (majority) electrons in the ferromagnet is faster than for spin-down. This sign agrees with results for tunneling from ferromagnets through $\mathrm{Al}_{2} \mathrm{O}_{3}$ into thinfilm superconducting Al (FIS devices) [10 16], although the sign is opposite to the polarization of the DOS at the Fermi level within band structure calculations [17]. This is understood to be due to much larger tunneling matrix elements for predominantly $s p$-band majority-spin electrons compared to predominantly $d$-band minority electrons, so that the matrix elements dominate over the DOS effect in determining the relative tunneling rates [18, 19]. The magnitude of the tunneling polarization that we measure $(8-12 \%)$ is considerably less than the values $35-42 \%$ found for Co using FIS devices [10,16], and we observe some field dependence not seen in larger samples (Fig. 3(d)). Both effects may indicate imperfections 
in our tunnel barriers; they have not undergone the process of optimization which achieved large polarizations in larger-area devices 20]. The presence of any oxidation at the magnetic interface can reduce the tunneling polarization 20. Our barriers are also very thin (with resistancearea products less than $200 \Omega-\mu \mathrm{m}^{2}$ compared to $10^{7}-10^{10}$ $\Omega-\mu \mathrm{m}^{2}$ in most prior experiments [10,21]), which might reduce the polarization by increasing the relative tunneling rate of $d$-states [22]. (However, recent work on optimized large-area $\mathrm{F} / \mathrm{Al}_{2} \mathrm{O}_{3} / \mathrm{F}$ tunnel junctions with $R A \sim 100 \Omega-\mu \mathrm{m}^{2}$ does not show reduced polarization [21.) We have considered whether the magnetic electrode might enhance spin relaxation within the particle so that it should not be neglected. This cannot explain the full reduction in our polarization; treating the relaxation rate as a free parameter, the maximum polarization consistent with the current steps in Fig. 3(a) is $21 \%$.

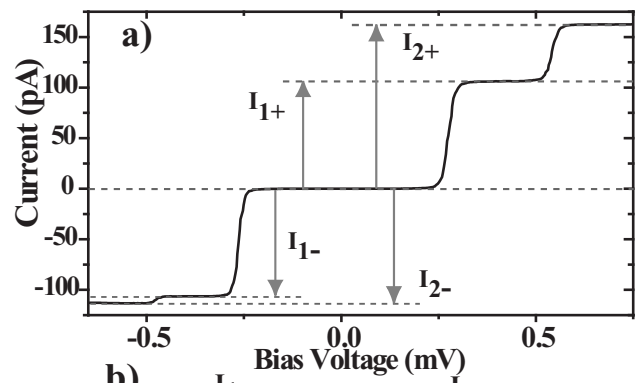

b)
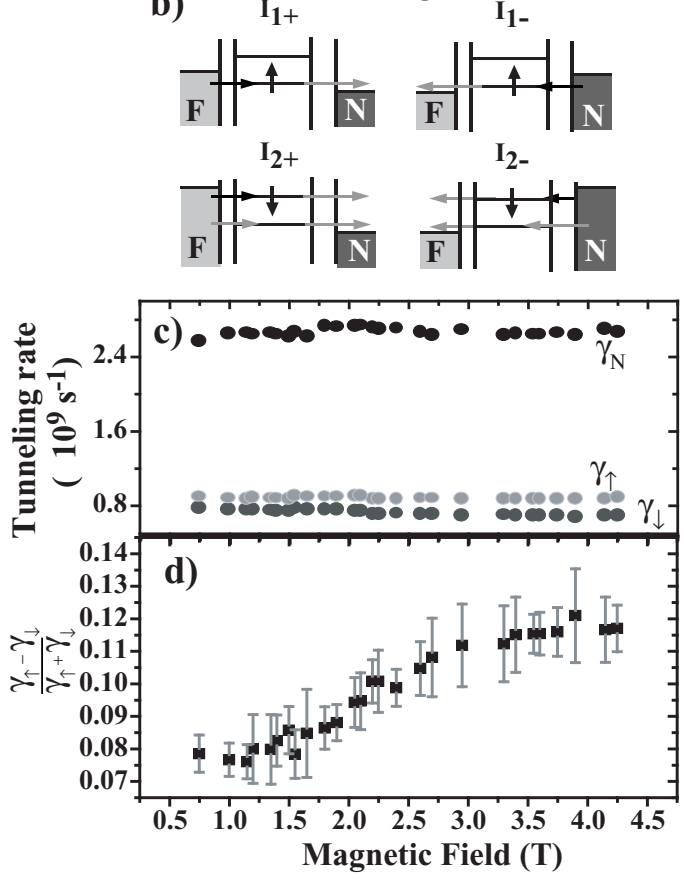

FIG. 3. (a) Current vs. voltage curve for device Co\#1 for $B=1 \mathrm{~T}$, showing the range of $V$ where tunneling occurs via one pair of Zeeman-split energy levels. (b) Energy-level diagrams at each current step. Black horizontal arrows show the threshold tunneling transition. Gray arrows depict other transitions which contribute to the current. (c) The tunneling rates $\gamma_{\uparrow}, \gamma_{\downarrow}$, and $\gamma_{N}$ determined as described in the text. (d) Tunneling polarization for device Co\#1.
In Figs. 1(b) and 2(c) at negative $V$, some higherenergy spin-down resonances produce $d I / d V<0$, meaning that they decrease the total current. This is a consequence of the slower rate of tunneling for minority-spin electrons; an electron in the spin-down state blocks current flow through the spin-up channel until the electron tunnels slowly to the F electrode. By incorporating additional states into the sequential-tunneling model, we find a tunneling polarization of $15 \pm 6 \%$ for the second Zeeman pair in sample $\mathrm{Co \# 1.}$

Let us now consider the $V$ positions of the tunneling resonances as a function of $B$. The magnitude of the Zeeman splitting is similar to previous measurements in all-Al devices [5]. After converting from $V$ to energy as described above, we determine the g-factor according to $\Delta E_{\text {Zeeman }}=g \mu_{B} B$. For the levels in Ni\#1, $g$ is between $1.83 \pm 0.05$ and $1.90 \pm 0.07$, in Co\#1 between $1.98 \pm 0.07$ and $2.05 \pm 0.06$, and in the other devices discussed in this paper, $1.9 \leq g \leq 2.0$. However, the data in Fig. 2 differ from studies with non-magnetic electrodes [5.15] in that the slopes of the spin-up and spindown Zeeman shifts are not symmetric about 0; in Figs. $2(\mathrm{a}, \mathrm{b})$ the midpoints of the Zeeman-split states tend to higher $|V|$ as a function of $B$ and in Fig. 2(c) they tend to lower values of $|V|$. This effect is expected in singleelectron transistors (SETs) made with magnetic components, as a result of a field-dependent change in a magnet's electrochemical potential 11, 12, . A related shift has been observed in micron-scale $\mathrm{Ni} / \mathrm{Co} / \mathrm{Ni}, \mathrm{Co} / \mathrm{Ni} / \mathrm{Co}$, and $\mathrm{Al} / \mathrm{Co} / \mathrm{Al}$ SETs [11]. When a magnetic field is applied to any bulk metal, it will flip some electron spins to align with $B$. Because a ferromagnet has different densities of states at the Fermi level for majority- and minorityspin states, the electrochemical potential must shift with $\mathrm{B}$ to accommodate the flipped spins. The magnitude of the shift will also be enhanced by exchange interactions in the magnet 12. We will parameterize the shift by the variable $S$, such that for an isolated magnetic sample $\Delta E_{F}(B)=S \mu_{B} B$. When a magnet is incorporated as one electrode in an otherwise nonmagnetic SET, the experimental consequences of this shift are equivalent to a change in the energy of all the states in the nanoparticle by the amount $d E / d B=-\mu_{B} S C_{F} /\left(C_{N}+C_{F}\right)$ [11]. This analysis assumes that the magnetic field does not induce any rearrangements of charge density (see below).

Within each sample, the average slopes of the different Zeeman-split pairs correspond to the same value of $S$ within measurement uncertainty. For the data in Figs. 2(a,b), the average slopes are $(2.6 \pm 0.2) \times$ $10^{-2} \mathrm{mV} /$ Tesla for positive $V$ and $(1.85 \pm 0.2) \times 10^{-2}$ $\mathrm{mV} /$ Tesla for negative $V$, giving in both cases $S=$ $0.45 \pm 0.04$. For non-interacting electrons with different majority and minority densities of states per unit energy at the Fermi level, $\rho_{\uparrow}$ and $\rho_{\downarrow}$, the DOS polarization would give a shift $S=-(1 / 2) g\left[\left(\rho_{\uparrow}-\rho_{\downarrow}\right) /\left(\rho_{\uparrow}+\rho_{\downarrow}\right)\right]$, where $g$ is the $g$-factor $[11,12$. Therefore a positive sign for $S$ corre- 
sponds to a greater density of minority-spin states at the Fermi level, in agreement with band structure calculations for $\mathrm{Ni}$ and $\mathrm{Co}$ [17]. However, the magnitude of the measured shift is surprisingly small. Band-structure calculations for Ni give $\rho_{\downarrow} / \rho_{\uparrow}=8.5$ [17], so that one would expect $S>0.79$. We write this as a lower limit, because exchange interactions should increase $S$ relative to predictions for non-interacting electrons [12]. For two other devices with a Ni electrode, made by the same procedure, we find even more striking discrepancies: $S=0.15 \pm 0.1$ and $0.2 \pm 0.1$. For 3 samples with a Co electrode, for which band structure calculations suggest that $S>0.59$ [17, we observe $S=0.1 \pm 0.1,0.37 \pm 0.05$ (for Co\#1), and $0.7 \pm 0.1$. Even though the electrochemical shift is expected to be a bulk property of the magnet 11, 12, we find significant sample-to-sample variations for the shift in the magnet's electrochemical potential relative to the Al particle.

We propose that the explanation of these discrepancies is that a magnetic field may produce rearrangements in the charge distribution inside a magnetic tunnel junction that will shift the energy levels of the particle as a function of $B$, with different strengths in each device. The predominantly $d$-band character of the minority electrons in $\mathrm{Co}$ or $\mathrm{Ni}$ will cause their wavefunctions to decay over a shorter distance as they penetrate into the tunnel barrier than for the predominantly $s p$-band majority electrons 22. Therefore, as an applied magnetic field transfers electrons from minority to majority states, some charge density at the surface of the magnet should shift slightly toward the barrier region [23. More complicated spindependent interface states could act similarly. The sign of the effect should cause the measured values of $S$ to decrease for $\mathrm{Ni}$ and Co electrodes, and the magnitude will be given by the work that the moving charge does on an electron in the particle. Making the approximation that the spin-dependent densities at the magnet's surface are similar to the bulk, the charge density per unit area which changes spin at the last monolayer of the magnet is $\sigma \approx e a \rho_{\uparrow} \rho_{\downarrow} g \mu_{B} B /\left(\rho_{\uparrow}+\rho_{\downarrow}\right)$, where $a$ is the lattice constant. If the average position for charges in the minority and majority states differs by $\Delta x$ at the surface layer, then this charge movement should change the measured electrochemical shift by

$$
\begin{aligned}
\Delta S & \approx-\frac{e^{2}}{\epsilon_{0}} g a(\Delta x) \frac{\rho_{\uparrow} \rho_{\downarrow}}{\rho_{\uparrow}+\rho_{\downarrow}} \\
& \approx-12 \Delta x / \AA
\end{aligned}
$$

for either a Co or Ni electrode. Therefore even in micronscale devices [11], $\Delta x$ as small as $0.01 \AA$ may decrease $S$ by $10 \%$, and foil attempts to measure the DOS polarization. In our devices, which have possibly non-uniform tunnel barriers, variations in $\Delta x$ by less than $0.05 \AA$ can explain the sample-to-sample differences.

In summary, we have studied tunneling between a bulk ferromagnet and the spin-resolved energy levels in a quantum dot. The energy levels can be used as spin filters, permitting a measurement of the different tunneling rates from the magnet for spin-up and spin-down electrons. As a function of $B$, the electrochemical potential in the magnet shifts relative to the energy levels in the quantum dot. In addition to the shift that is expected due to the magnet's bulk density-of-states polarization [11], we suggest that there is an important contribution from $B$-dependent redistributions of charge at the magnetic interface.

We thank R. A. Buhrman, A. Champagne, S. Guéron, A. H. MacDonald, D. J. Monsma, and E. B. Myers for discussions. Funding was provided by ARO (DAAD1901-1-0541), the Packard Foundation, and NSF (DMR0071631 and use of the National Nanofabrication Users Network).

[1] P. L. McEuen et al., Phys. Rev. Lett. 66, 1926 (1991).

[2] S. Tarucha et al., Phys. Rev. Lett. 77, 3613 (1996).

[3] D. R. Stewart et al., Science 278, 1784 (1998).

[4] M. Ciorga et al., Phys. Rev. B 61, R16315 (2000).

[5] D. C. Ralph, C. T. Black, and M. Tinkham, Phys. Rev. Lett. 74, 3241 (1995).

[6] J. R. Petta and D. C. Ralph, Phys. Rev. Lett. 87, 266801 (2001).

[7] D. H. Cobden et al., Phys. Rev. Lett. 81, 681 (1998).

[8] S. Guéron, M. M. Deshmukh, E. B. Myers, and D. C. Ralph, Phys. Rev. Lett. 83, 4148 (1999); M. M. Deshmukh et al., Phys. Rev. Lett. 87, 226801 (2001).

[9] P. Recher, E. V. Sukhorukov, and D. Loss, Phys. Rev. Lett. 85, 1962 (2000).

[10] R. Meservey and P. M. Tedrow, Phys. Rep. 238, 173 (1994).

[11] K. Ono, H. Shimada, and Y. Ootuka, J. Phys. Soc. Jpn. 66, 1261 (1997); 67, 2852 (1998).

[12] A. H. MacDonald, in Proceedings of the XVI Sitges Conference on Statistical Mechanics (Springer-Verlag, Berlin, 2000), p. 211.

[13] J. von Delft and D. C. Ralph, Phys. Rep. 345, 61 (2001).

[14] E. Bonet, M. M. Deshmukh, and D. C. Ralph, Phys. Rev. B 65, 045317 (2002).

[15] M. M. Deshmukh, E. Bonet, A. N. Pasupathy, and D. C. Ralph, Phys. Rev. B 65, 073301 (2002).

[16] D. J. Monsma and S. S. P. Parkin, Appl. Phys. Lett. 77, $720(2000)$.

[17] D. A. Papaconstantopoulos, Handbook of the Band Structure of Elemental Solids (Plenum, NY, 1986).

[18] M. B. Stearns, J. Magn. Magn. Mater. 5, 167 (1977).

[19] E. Y. Tsymbal and D. G. Pettifor, J. Phys.: Condens. Matter 9, L411 (1997).

[20] L. Seve et al., Europhys. Lett. 55, 439 (2001).

[21] D. J. Monsma and S. S. P. Parkin, unpublished.

[22] I. I. Oleinik, E. Y. Tsymbal, and D. G. Pettifor, Phys. Rev. B 62, 3952 (2000).

[23] A related effect has been considered by S. Zhang, Phys. Rev. Lett. 83, 640 (1999). 